XX Congreso de Ecuaciones Diferenciales y Aplicaciones

X Congreso de Matemática Aplicada

Sevilla, 24-28 septiembre 2007

(pp. 1-8)

\title{
An optimal design problem in wave propagation
}

\author{
J.C. Bellido ${ }^{1}, \underline{\text { A. Donoso }}^{1}$ \\ ${ }^{1}$ Dpto. Matemáticas (ETSII), Universidad de Castilla la Mancha, Edificio Politécnico, Avda. Camilo \\ José Cela s/n, 13071, Ciudad Real. E-mails: JoseCarlos.Bellido@uclm.es, Alberto.Donoso@uclm.es.
}

Palabras clave: Optimal design, wave propagation, classical solutions

\section{Resumen}

In this paper we consider an optimal design problem in wave propagation proposed in [1] in the one-dimensional situation: Given two materials at our disposal with different Young's modulus and different density, the problem consists of finding the best distributions of the two initial materials in a rod in order to minimize the vibration energy in the structure under periodic loading of driving frequency $\Omega$. We comment on relaxation and optimality conditions, and perform numerical simulations of the optimal configurations. We also prove the existence of classical solutions in certain cases.

\section{Introduction}

In [1] a structural model was proposed for the design of finite structures made up of two given materials in the context of wave propagation. The model is an optimal design problem in which the distribution of two given materials is optimized so as to minimize a cost functional related to the vibration or propagation of waves along the medium. In practice, this model may be useful for the systematic design of wave filters, damping of waves, or wave guides. The authors develop a numerical method for the optimization of those structures based on topology optimization (see [2]), but there is no mathematical analysis of the model. In the numerical examples, they also observe the surprising fact that no microstructure appears between the two materials when one tries to design a filter or to minimize the vibration energy. Our aim here is to analyze mathematically the model proposed in [1] in the one-dimensional situation for longitudinal propagation.

We consider the one-dimensional wave equation

$$
\rho w_{t t}=\left(E w_{x}\right)_{x}
$$


where $t$ is the time variable, $x$ the space variable, $w$ is the displacement, and $E$ and $\rho$ are the Young's modulus and material density respectively. Notice we do not consider any damping term in our equation. We are interested in time-harmonic solutions under periodic loading of the previous equation, i.e. in solutions of the form

$$
w(x, t)=u(x) \exp (i \Omega t)
$$

where $\Omega$ is the driving frequency and $u$ the amplitude function. Then the amplitude, $u$, verifies the equation

$$
\left(E(x) u^{\prime}\right)^{\prime}+\Omega^{2} \rho(x) u=0 .
$$

Now, let us assume that we have two materials at our disposal, and consequently the elastic coefficient, $E(x)$, and the density, $\rho(x)$, take two values on, $E_{1}, E_{2}\left(0<E_{1}, E_{2}\right)$ and $\rho_{1}, \rho_{2}\left(0<\rho_{1}, \rho_{2}\right)$ respectively. We make the technical assumption

$$
E_{1} \leq E_{2}, \quad \rho_{1} \leq \rho_{2}
$$

and that at least one of the inequalities is strict (otherwise we would just have one material). If we have a rod of length $l$, that we represent by the interval $[0, l]$, and we distribute the two materials in the rod, the elastic coefficient function and the density function are respectively

$$
E(x)=E_{1} \chi(x)+E_{2}(1-\chi(x)), \quad \rho(x)=\rho_{1} \chi(x)+\rho_{2}(1-\chi(x)),
$$

where $\chi$ is the characteristic function of the (measurable) subset of the rod where we place the first material. Now it is natural to consider the following optimal design problem,

$$
\operatorname{Min}_{\mathcal{A}_{a d}} J(\chi),
$$

where the admissible set of designs is

$$
\mathcal{A}_{a d}=\{\chi: \text { characteristic function of a measurable subset of }[0, l]\},
$$

the cost functional is the vibration energy

$$
J(\chi)=\int_{0}^{l}\left[\Omega^{2}\left(\rho_{1} \chi(x)+\rho_{2}(1-\chi(x))\right)|u(x)|^{2}+\left(E_{1} \chi(x)+E_{2}(1-\chi(x))\right)\left|u^{\prime}(x)\right|^{2}\right] d x,
$$

and $u$ is computed from $\chi$ through the state equation

$$
\begin{aligned}
& {\left[\left(E_{1} \chi(x)+E_{2}(1-\chi(x))\right) u^{\prime}\right]^{\prime}+\Omega^{2}\left(\rho_{1} \chi(x)+\rho_{2}(1-\chi(x))\right) u=0,} \\
& \left(E_{1} \chi(0)+E_{2}(1-\chi(0))\right) u^{\prime}(0)=\gamma, \quad\left(E_{1} \chi(l)+E_{2}(1-\chi(l))\right) u^{\prime}(l)=0,
\end{aligned}
$$

$\gamma$ is a fixed given nonzero number. Here we consider the case of boundary conditions corresponding to one external load on the left extreme of the rod, and homogeneous Neumann boundary conditions on the other extreme. Roughly speaking, the optimal design problem consists of determining the best distribution (i.e., the best $\chi$ ) of the two given materials in the rod in order to minimize the vibration energy in the structure, so that we minimize the vibration amplitude along the rod. Notice that our optimal design problem indeed is an optimal design problem for an ODE, as we have simplified the wave equation by considering time harmonic solutions. 
We do not consider any volume constraint in this problem, and this is in accordance with the physical nature of the problem since waves propagate better through homogeneous materials than through mixtures of two materials. However the problems makes perfect sense if we impose a restriction on the amount of the materials that we may use, as for instance the constraint

$$
\int_{0}^{l} \chi(x) d x \leq \delta, \text { or } \int_{0}^{l} \chi(x) d x=\delta,
$$

with $\delta \in(0, l)$ a fixed constant.

The solutions of the state equations are understood as weak solutions. That equation is obviously nonelliptic; however by the Fredholm's alternative, we can claim the existence of a unique solution for any boundary data if and only if $\Omega^{2}$ is not an eigenvalue for the problem

$$
\begin{gathered}
-\left[\left(E_{1} \chi(x)+E_{2}(1-\chi(x))\right) u^{\prime}\right]^{\prime}=\lambda\left(\rho_{1} \chi(x)+\rho_{2}(1-\chi(x))\right) u, \quad u \in H^{1}(0, l), \\
\left(E_{1} \chi(0)+E_{2}(1-\chi(0))\right) u^{\prime}(0)=0, \\
\left(E_{1} \chi(l)+E_{2}(1-\chi(l))\right) u^{\prime}(l)=0 .
\end{gathered}
$$

Recall that, as the operator involved is compact and self-adjoint, we know that there exists an increasing (maybe not strictly) sequence of eigenvalues. For the sake of simplicity, and in order to formulate the optimal design problem, we will assume that $\Omega^{2}$ is far away of any eigenvalue of the problem (1a-1c) for any characteristic function $\chi$. This hypothesis makes physical sense (see [1]).

It is well-known that in principle we cannot hope the previous optimal design problem to admit optimal solutions, and in general we need to study relaxation for it (see [4] and the references therein).

The plan of the paper is the following. Section 2 is devoted to state a relaxation result for our optimal design problem and to give necessary conditions of optimality for the relaxed formulation of the problem. In Section 3 we obtain qualitative properties of the optimal solutions of the relaxed problem, being able to prove, in certain cases, that there are optimal solutions in the form of characteristic functions, what implies the existence of minimizers for the original optimal design problem (P). Finally, in Section 4 we perform numerical simulations by using a gradient method based on the sensitivities computed in Section 2 and analyze numerically examples of practical interest.

\section{Relaxation}

Relaxation for optimal control or optimal design problems for ODE's is a classical subject. In this sense our optimal design problem fits into this framework and relaxation for it is straightforward, and so to prove the relaxation result stated below is standard (basic references on the subject are $[4,5,6]$ ).

Theorem 2.1 The optimal design problem

$$
\operatorname{Min}_{\mathcal{A}_{a d}^{\star}} \tilde{J}(E(\theta), \rho(\theta)),
$$


where the admissibility set is

$$
\mathcal{A}_{a d}^{\star}=\left\{\theta \in L^{\infty}(0, l): 0 \leq \theta \leq 1\right\},
$$

$E(\theta)$ and $\rho(\theta)$ are given by

$$
E=E(\theta)=E_{1} E_{2} /\left(E_{1}+\theta\left(E_{2}-E_{1}\right)\right), \quad \rho=\rho(\theta)=\rho_{1} \theta+\rho_{2}(1-\theta),
$$

the cost functional is

$$
\tilde{J}(E, \rho)=\int_{0}^{l}\left[\Omega^{2} \rho(x)|u(x)|^{2}+E(x)\left|u^{\prime}(x)\right|^{2}\right] d x,
$$

and $u$ is computed from the pair $(E, \rho)$ through the state equation

$$
\begin{gathered}
{\left[E u^{\prime}\right]^{\prime}+\Omega^{2} \rho u=0,} \\
E(0) u^{\prime}(0)=\gamma, \quad E(l) u^{\prime}(l)=0
\end{gathered}
$$

is a relaxation of the optimal design problem $(\mathrm{P})$.

We will need for the numerical simulations the gradient of the cost functional $\tilde{J}$ in the relaxed problem $(\tilde{\mathrm{P}})$. Its computation is direct by using the classical adjoint method.

Theorem 2.2 The functional $\tilde{J}$ in problem $(\tilde{\mathrm{P}})$ is Gâteaux differentiable on the set $\mathcal{A}_{a d}^{\star}$, and the Gâteaux derivative at $\bar{\theta}$ in the direction of the admissible variation $\theta$ (admissible variations means that $\theta \in L^{\infty}(0, l)$ such that there exists $h_{0}>0$ such that for every $\left.h \leq h_{0}, \bar{\theta}+h \theta \in \mathcal{A}_{a d}^{\star}\right)$ is given by

$$
\begin{array}{r}
\tilde{J}^{\prime}(\bar{\theta} ; \theta)=\int_{0}^{l}\left\{\left[E^{\prime}(\bar{\theta}(x))\left(\bar{u}^{\prime}(x) \bar{p}^{\prime}(x)+\left|\bar{u}^{\prime}(x)\right|^{2}\right)\right.\right. \\
\left.+\Omega^{2}\left(\rho_{2}-\rho_{1}\right)\left(\bar{u}(x) \bar{p}(x)-|\bar{u}(x)|^{2}\right)\right] \theta(x) \\
\left.-\left(E^{\prime}(\bar{\theta}(x)) \bar{u}(x) \bar{p}(x) \theta(x)\right)^{\prime}\right\} d x,
\end{array}
$$

where $\bar{u}$ is the state associated to $\bar{\theta}$ (i.e. the solution of $(2 \mathrm{a}-2 \mathrm{~b})$ for the pair $(E(\bar{\theta}) \rho(\bar{\theta}))$ ), and $\bar{p}$ is the adjoint state, being in this case the unique solution of

$$
\begin{gathered}
{\left[E(\bar{\theta}) \bar{p}^{\prime}\right]^{\prime}+\Omega^{2} \rho(\bar{\theta}) \bar{p}=2\left(\Omega^{2} \rho(\bar{\theta}) \bar{u}-\left[E(\bar{\theta}) \bar{u}^{\prime}\right]^{\prime}\right),} \\
E(\bar{\theta}(0)) \bar{p}^{\prime}(0)=-2 \gamma, \quad E(\bar{\theta}(l)) \bar{p}^{\prime}(l)=0 .
\end{gathered}
$$

Using the Taylor expansion of the cost functional we obtain, as a direct consequence of the previous result, the following maximum principle.

Corollary 2.1 If $\bar{\theta}$ (with $\bar{\theta}$ an element of the interior of $\mathcal{A}_{a d}^{\star}$ ) is an optimal solution for problem $(\tilde{\mathrm{P}})$ then

$$
\tilde{J}^{\prime}(\bar{\theta} ; \theta-\bar{\theta}) \geq 0
$$

for any $\theta$ such that $\theta-\bar{\theta}$ is an admissible variation. 


\section{Existence of Solutions for Problem (P)}

In this section we explore the consequences of the optimality conditions given in the form of a maximum principle in Corollary 2.1 on the optimal solutions. Our objective is to check whether it is possible to prove existence results for the original optimal design problem $(\mathrm{P})$. In the case that $E_{1}=E_{2}$ (and we assume then that $E_{1}=E_{2}=1$ ), the design variable does not act on the principle part of the state equation and the problem is mathematically much simpler, we are able to prove that indeed any optimal solution for problem $(\tilde{\mathrm{P}})$ is a characteristic function, so that the original optimal design problem admits minimizers. We use the optimality conditions in this particular case to show the result, which is given in Theorem 3.1. The general situation is more difficult and does not seem possible to use the same argument, however we give a condition implying the existence of at least one optimal solution in the form of a characteristic function. We divide this section into two subsections corresponding to the two cases.

\subsection{Case $E_{1}=E_{2}$}

We assume that $E_{1}=E_{2}=1$ and our problem now, that we denote as $\left(\tilde{\mathrm{P}}_{1}\right)$, is the following

$$
\operatorname{Min}_{\mathcal{A}_{a d}^{\star}} \tilde{J}(\rho(\theta)),
$$

where the admissibility set is

$$
\mathcal{A}_{a d}^{\star}=\left\{\theta \in L^{\infty}(0, l): 0 \leq \theta \leq 1\right\},
$$

$\rho(\theta)$ is given by

$$
\rho(\theta)=\rho_{1} \theta+\rho_{2}(1-\theta),
$$

the cost functional is

$$
\tilde{J}(\theta)=\int_{0}^{l}\left[\Omega^{2} \rho(\theta(x))|u(x)|^{2}+\left|u^{\prime}(x)\right|^{2}\right] d x,
$$

and $u$ is computed from $\theta$ through the state equation

$$
\begin{gathered}
{\left[u^{\prime}\right]^{\prime}+\Omega^{2} \rho(\theta(x)) u=0,} \\
u^{\prime}(0)=\gamma, \quad u^{\prime}(l)=0
\end{gathered}
$$

Following Theorem 2.1, this problem is in the relaxed form and therefore it admits at least one optimal solution. The following result establishes that any optimal solution is actually a classical one (classical solution means a solution in the form of a characteristic function).

Theorem 3.1 Any optimal solution, $\bar{\theta}$, for problem $\left(\tilde{\mathrm{P}}_{1}\right)$ takes on only the values $\{0,1\}$, i.e. any optimal solution for $\left(\tilde{\mathrm{P}}_{1}\right)$ is a characteristic function. 


\subsection{General case}

The existence of classical solutions for the general situation of $(\tilde{\mathrm{P}})$ is not clear in general as far as the authors are able to claim, although something may be said about this. We do not state a formal result in this section, but show using new ideas that the problem admits optimal solutions in certain cases. All the technical details are included in [3], but we give numerical evidence of this in the next section.

\section{Numerical Approach and Examples}

After the analysis carried out in the previous sections, this section is devoted to show several numerical simulations for our problem. The approach that we follow in the present work is to approximate directly the optimal solutions for the relaxed optimal design problem $(\tilde{\mathrm{P}})$ by a gradient method. Of course this implies approximation of the states and adjoint states, i.e. solutions of the homogenized state equation (see Theorem 2.1) and the corresponding adjoint equation (see Theorem 2.2). We believe that this approach will certainly give good approximations of the optimal designs for problem $(\tilde{\mathrm{P}})$.

The numerical algorithm used in this work is a variable step-size descent method given by

$$
\theta_{k+1}=\theta_{k}-\tilde{J}^{\prime}\left(\theta_{k}\right) s_{k},
$$

where $s_{k}$ is a small positive step such that $\tilde{J}\left(\theta_{k+1}\right)<\tilde{J}\left(\theta_{k}\right), \tilde{J}^{\prime}\left(\theta_{k}\right)$ is the gradient of the cost functional respect to the control, computed in Section 2, and the step $s_{k}$ is looked for as the step that minimizes $\tilde{J}\left(\theta_{k+1}\left(s_{k}\right)\right.$ ) (we use a descent method for computing $s_{k}$ ). On the other hand, since the control must verify that $0 \leq \theta_{k+1} \leq 1$, then the updating formula can be easily compacted by

$$
\theta_{k+1}=\operatorname{máx}\left(0, \operatorname{mín}\left(1, \theta_{k}-\tilde{J}^{\prime}\left(\theta_{k}\right) s_{k}\right)\right),
$$

being the same that we obtained by using a projected gradient method. Concerning the stopping criteria, it has been checked that the cost functional slightly changes its value and therefore, the $L^{\infty}$-norm of the difference $\tilde{J}\left(\theta_{k+1}\right)-\tilde{J}\left(\theta_{k}\right)$ reduces to zero, as we approximate to the minimum. All numerical examples belonging to this work have been obtained under the stopping criteria, tol $=10^{-5}$, requiring for convergence about 15 iterations of the algorithm, on average. It has also been tested that very similar results are obtained when we run the same numerical examples with different mesh sizes.

For the sake of briefness, we illustrate our approach through two numerical examples. In both of them, the design domain is an elastic rod of unit length subjected to time harmonic longitudinal excitation of amplitude $\gamma$ in the left end. Material properties are $E_{2}=200 \mathrm{GPa}, \rho_{2}=7,800 \mathrm{~kg} / \mathrm{m}^{3}$, and $E_{1}=70 \mathrm{GPa}, \rho_{1}=2700 \mathrm{~kg} / \mathrm{m}^{3}$ corresponding to steel and aluminum, respectively. In previous sections it has been possible to prove that optimized solutions are classical when the Young's modulus $E$ keeps constant along the rod and to show that there are cases with $E$ varying its value in which still there are classical solutions. In general, numerical simulations indicate that optimized layouts are classical, and therefore, there is no microstructure among the two phases we are mixing.

In the first example we have used the frequency value $\Omega=50 \mathrm{kHz}$. Even though we let the control $\theta$ vary continuously between 0 and 1 during the optimization process, we can 
see in Figure 1(a) that the optimized solution obtained is classical at the end of the process. Notice that this configuration make the wave damp itself in an optimized way, as it travels along the rod (see Figure 1(b)). Similar conclusions as before can be obtained in the second example for $\Omega=200 \mathrm{kHz}$ (see Figure 1(c) and (d)). We can repeat the same numerical examples but keeping constant the Young's modulus, for instance, $E_{2}=E_{1}=70 \mathrm{GPa}$. The numerical results for $\Omega=50 \mathrm{kHz}$ and $200 \mathrm{kHz}$ (Figure 2) corroborate the existence of classical solutions such as it was analytically proved.

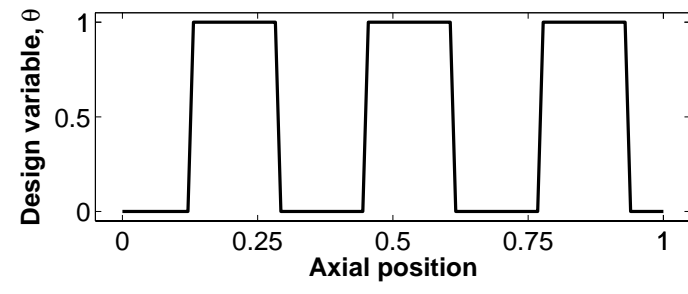

(a) Optimized design

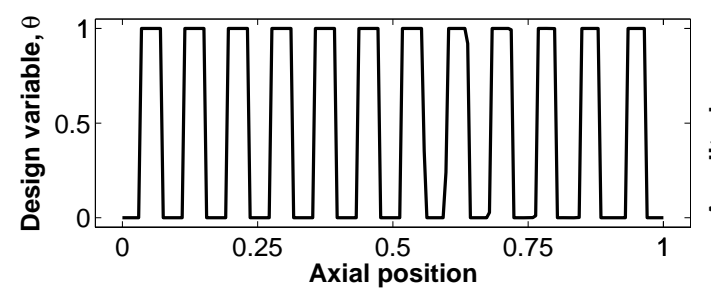

(c) Optimized design

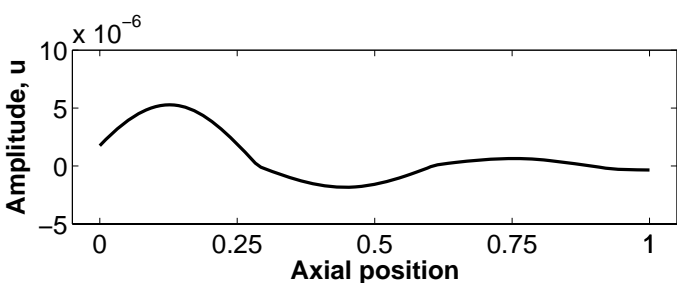

(b) Wave amplitude

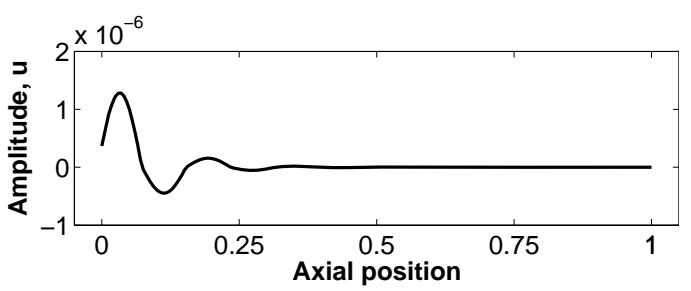

(d) Wave amplitude

Figura 1:

It is important to notice that (for this particular case $E_{2}=E_{1}$ ) we initially tried with a numerical approach based on optimality conditions. Although in some situations such algorithm gave us the same results as the ones obtained by the gradient method, the fact is that it did not enjoy good convergence properties in general, but when it did we got similar results.

In view of the numerical results we can state that, even though the design problem treated is this paper is rather different to the band-gap one, as we commented in the Introduction, the optimized finite structures (almost periodic in some cases) obtained (for minimum vibration energy) for a particular value of $\Omega$, not avoid (like in the band-gap situation) but make rather difficult the wave can propagate itself along the rod only at that corresponding value of $\Omega$.

To conclude, it is worth emphasizing that first, all this analysis makes sense for high frequency values (typically greater than $10 \mathrm{kHz}$ ), otherwise the optimal designs are "boring" because they would correspond to take only the material with higher ratio $\left(E_{i} / \rho_{i}\right)$, for $i=1,2$, and second, the same layouts we present here are obtained whenever the product $\Omega l$ keeps constant. 


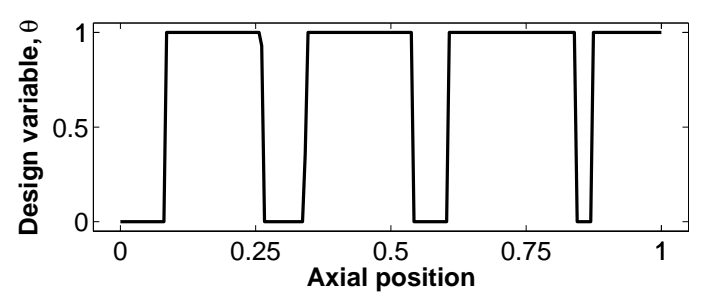

(a) Optimized design

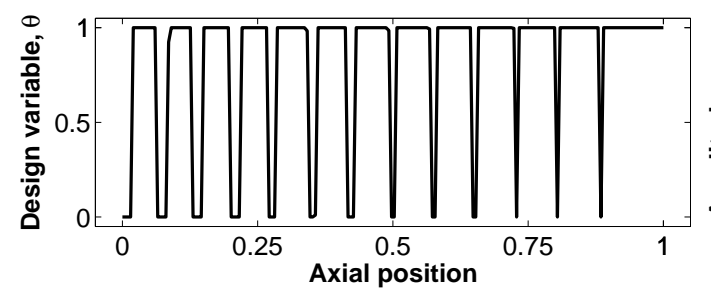

(c) Optimized design

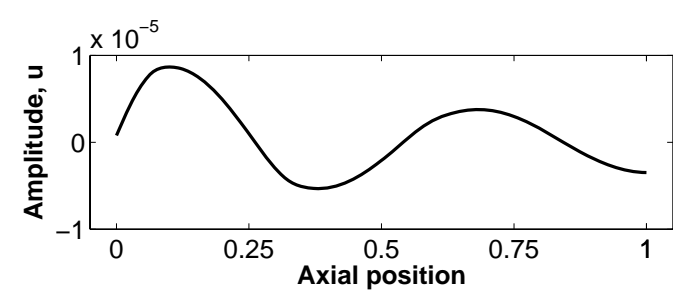

(b) Wave amplitude

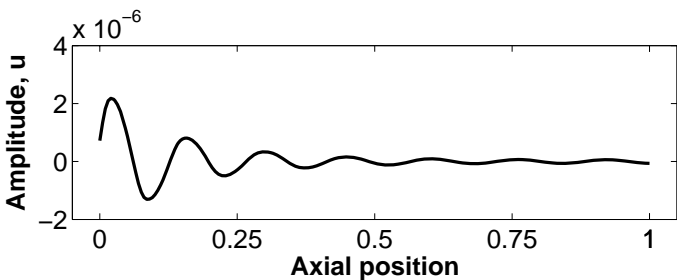

(d) Wave amplitude

Figura 2:

\section{Acknowledgement}

The authors acknowledge many stimulating discussions on the subject of this paper with Martin Bendsøe, Jacob Jensen and Ole Sigmund. Financial support was provided by MEC (Spain) Grant MTM2004-07114, and by Junta de Comunidades de Castilla-La Mancha (Spain) Grant PAI-05-027.

\section{Referencias}

[1] SIGMUND O. and JENSEN J.S. Systematic Design of Phononic Band-Gap Materials and Structures by Topology Optimization, The Royal Society of London, Philosophical Transactions, Series A, Vol. 361, pp. 1001-1019, 2003.

[2] BENDSøE M.P. and SIGMUND O. Topology Optimization: Theory, Methods and Applications, Springer-Verlag, Berlin, Germany, 2003.

[3] BELLIDO J.C. and DONOSO A. An optimal design problem in wave propagation, to appear in Journal of Optimization Theory and Applications.

[4] LURIE K.A. Applied Optimal Control Theory of Distributed Systems, Plenum Press, New York, New York, 1993.

[5] WARGA J. Optimal Control of Differential and Functional Equations, Academic Press, New York, New York, 1972.

[6] YOUNG L.C. Lectures on the Calculus of Variations and Optimal Control Theory, W. B. Saunders Coorporation, Philadelphia, Pennsylvania, 1969. 